\title{
Fast surface segmentation guided by user input using implicit extension of minimal paths
}

\author{
Roberto Ardon ${ }^{1,2}$, Laurent D. Cohen ${ }^{2}$, and Anthony Yezzi ${ }^{3}$ \\ 1 MEDISYS-Philips France, \\ 51, rue Carnot, 92156 Suresnes, France \\ 2 CEREMADE, UMR CNRS 7534, Université Paris Dauphine, \\ Place du Marchal de Lattre de Tassigny, 75775 Paris Cedex 16, France \\ 3 Georgia Institute of Technology, Atlanta, GA, USA \\ Email: roberto.ardon@philips.com,cohen@ceremade.dauphine.fr, ayezzi@ece.gatech.edu
}

\begin{abstract}
We introduce a novel implicit approach for single object segmentation in 3D images. The boundary surface of this object is assumed to contain two or more known curves (the constraining curves), given by an expert. The aim of our method is to find the desired surface by exploiting the information given in the supplied curves as much as possible. We use a cost potential which penalizes image regions of low interest (for example areas of low gradient). In order to avoid local minima, we introduce a new partial differential equation and use its solution for segmentation. We show that the zero level set of this solution contains the constraining curves as well as a set of paths joining them. These paths globally minimize an energy which is defined from the cost potential. Our approach, although conceptually different, can be seen as an implicit extension to $3 D$ of the minimal path framework already known for $2 D$ image segmentation. As for this previous approach, and unlike other variational methods, our method is not prone to local minima traps of the energy. We present a fast implementation which has been successfully applied to 3D medical and synthetic images.
\end{abstract}

Keywords: Image segmentation, Active contours, Minimal Paths, Level Set method, Object Extraction, Stationary Transport Equation.

\section{Introduction}

The common use of deformable models, introduced by Kass et al. [18], in 2D and 3D image segmentation consists of introducing an initial object in the image and deforming it until it reaches a desired target. Generally, the evolution of the object is chosen in order to most rapidly reduce an energy involving the image data until a steady state is reached. One of the main drawbacks of this approach is that it suffers from local minima 'traps'. This is the case when the steady state reached by the active object does not correspond to the target but to another local minimum of the energy. As a consequence, the active object's initialization is of crucial importance: the final result depends strongly upon it. 
Since the publication of [18], much work has been done in order to free active models from the problem of local minima. A balloon force was proposed in [9], making the model more active, driving it over 'weak' energy minima toward deeper ones, hopefully corresponding to better segmentations. Nonetheless this force assumes a known direction in the evolution (expanding or shrinking) and does not ensure pertinence of the reached minimum.

Other improvements have been achieved through the introduction of region dependent [10,30,23] and shape prior energies [29]. Their use has a smoothing effect on the global energy, decreasing the number of non-significant local minima and thus giving a more robust model. However these energies are often specific to the treated problem and much more information, other than the original image, has to be introduced.

In [13], Cohen and Kimmel present a segmentation approach in 2D where the final state of the active curve stands for the global minimum of an image dependent energy. Their model only requires two points located on the boundary to be segmented as additional information. Unfortunately their approach cannot be extended in a simple way to find the global minimum for an active surface in a 3D image. In some sense, this paper proposes an extension to surface segmentation.

In this work, we focus on a novel approach for 3D single object segmentation where the resulting surface globally minimizes a given energy. Our aim is to generate a surface that contains a couple of 'constraining' curves $\left(\Gamma_{1}\right.$ and $\left.\Gamma_{2}\right)$ and which is also a segmentation of a target object. $\Gamma_{1}$ and $\Gamma_{2}$ are assumed to be traced by an expert ${ }^{4}$ on the surface to be segmented. Our approach is based on implicitly generating a surface that contains the set of paths globally minimizing an image energy and connecting $\Gamma_{1}$ and $\Gamma_{2}$. Moreover, the constraining curves are the only input for the initialization of our model. The paths linking $\Gamma_{1}$ and $\Gamma_{2}$ are globally minimal with respect to an energy of the form $\int_{\Gamma} \widetilde{\mathcal{P}}$, where $\widetilde{\mathcal{P}}$ is a positive function defined on the image domain. If the incremental cost $\widetilde{\mathcal{P}}$ is chosen to take lower values on the contours of the 3D image, in particular on the surface of the object to be extracted, global minimal paths will help finding the boundary of the object (see section 2 and [13]). This fact has been exploited in previous work [3,4], where a network of a finite number of minimal paths was computed between the two constraining curves and then extended, by means of interpolation, to a segmenting surface of the object.

Although this approach gave good results, particularly in ultrasound 3D images, the topology of the network was often problematic (paths tend to merge and only a few points of $\Gamma_{1}$ are reached, see figure 1.d), considerably complicating the generation of the segmenting surface and in the worst cases leading to bad segmentations (figure 2.b).

Although based on similar ideas, our model is more than an extension of the network approach. The surface generated by our algorithm is completely composed of globally minimal paths ${ }^{5}$, and in particular, it contains all the minimal paths of the network introduced in [4] (no explicit computation of minimal paths is needed nor any

\footnotetext{
${ }^{4}$ notice that the expert may obtain these curves with a $2 \mathrm{D}$ active contour or interactive tool like with the minimal path approach in [16]

${ }^{5}$ Note that this is very different than the minimal surface (if it exists) between the bounding curves.
} 


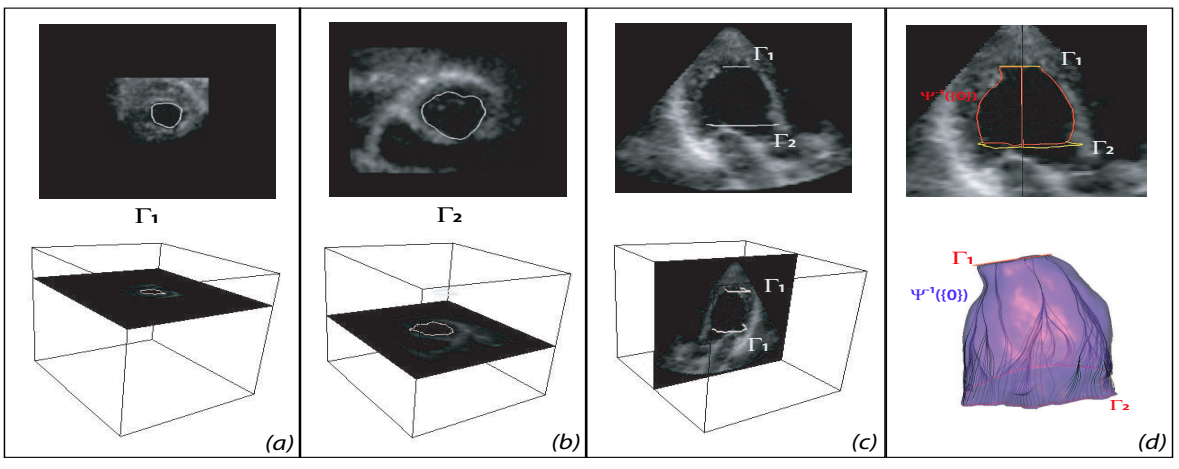

Fig. 1: 3D ultrasound volume of a left ventricle: (a) and (b) show the two parallel slices where the user given curves $\Gamma_{1}$ and $\Gamma_{2}$ are drawn. (c) shows a slice perpendicular to the curves in order to show their position with respect to the ventricle. Finally (d) shows the surface containing the constraint curves obtained with our approach. In the upper position we have shown the intersection of the zero level set of $\Psi$ with a vertical plane. In the lower position we have traced some minimal paths between the two constraining curves and a 3D representation of the zero level set, the minimal paths are traced on this surface.

interpolation method). Indeed, by solving a stationary transport equation of the form: $\nabla \Psi . \nabla U+G(\Psi)=0$, where $U$ is the action map (defined in section 3), $G$ is a regularization function and $\Psi$ is the unknown, we show in section 4 that $\Psi$ is such that: any minimal path between the constraining curves is contained in its zero level set (further noted $\left.\Psi^{-1}(\{0\})\right)$. More generally, we prove that this set is only composed of minimal paths: if a point belongs to $\Psi^{-1}(\{0\})$ then the globally minimal path joining this point to $\Gamma_{1}$ is contained as well in $\Psi^{-1}(\{0\})$. This property is the key point explaining the good performance of our algorithm. In section 5, we propose symmetrization of the approach and an extension to more than two given curves. In section 7 we give some results obtained by our method on synthetic and real data.

As an illustration of our problem, we give in figures 1(a), 1(b) and 1(c) an example of the user input to our algorithm. We perform the segmentation of a 3D ultrasound volume of the left ventricle. In figure 1(d) we show the output of our method, given by the zero level set of the function $\Psi$. We have also traced, for demonstration purposes, some minimal paths joining points of $\Gamma_{2}$ to $\Gamma_{1}$, which are clearly displayed on the segmenting surface.

\section{Minimal paths in 2D images}

\subsection{Active contour model}

The first active contour model was introduced by Kass et al. in the seminal paper [18]. Their model, the well known 'snakes', consists of finding a curve $\mathcal{C}$ (parametrized over the interval $[0,1]$ and traced on the image) that minimizes the energy, 


$$
E_{s}(\mathcal{C})=\int_{0}^{1}\left\{w_{1}\left\|C^{\prime}(u)\right\|^{2}+w_{2}\left\|C^{\prime \prime}(u)\right\|^{2}+\mathcal{P}(\mathcal{C}(u)) d u\right\}
$$

where $w_{1}$ and $w_{2}$ are positive constants. The minimization of the first two terms of $E_{s}$ induces the curve $\mathcal{C}$ to have high regularity, while the minimization of the latter is intended to drive the curve toward significant edges in the image. The potential function $\mathcal{P}$ usually represents an edge detector that has lower values along edges. A common choice for this function is

$$
\mathcal{P}=\frac{1}{1+|\nabla I|^{2}}
$$

if $I$ is the image. Finding a curve that minimizes the energy $E_{s}$ is not a simple task; this functional is defined on an infinite dimensional space and generally it is non-convex. The usual approach is to find a local minimum of $E_{s}$ by evolving an initial curve $\mathcal{C}_{0}$ under the time dependent equation: $\frac{\partial \mathcal{C}(u, t)}{\partial t}-w_{1} \frac{\partial^{2} \mathcal{C}(u, t)}{\partial u^{2}}+w_{2} \frac{\partial^{4} \mathcal{C}(u, t)}{\partial u^{4}}=-\nabla \mathcal{P}(\mathcal{C})$, with $\mathcal{C}(\cdot, 0)=\mathcal{C}_{0}$. Through this approach, the final curve $\mathcal{C}$ is strongly dependent on the initialization $\mathcal{C}_{0}$. Since the method was originally intended to interactively segment a single object in the image, this behavior is rather natural. Nonetheless, if $\mathcal{C}_{0}$ is too far from the object to extract, the evolving curve can become trapped in another local minimum, thus giving an unsatisfactory result.

\subsection{Active contours and minimal paths}

In order to obtain global minimization in the active contours framework, Cohen and Kimmel [13] simplified the energy by choosing $w_{2}=0$ in the expression (1)and used arclength parameterization (here noted $s$ ), thus exploiting the geometry of the curve and not its parametric description. They look for the curve minimizing the energy

$$
E(\mathcal{C})=\int_{0}^{L}\{w+\mathcal{P}(\mathcal{C}(s))\} d s
$$

where $w>0$ is a constant and $\mathcal{P} \geq 0$ is defined on the image domain, $L$ is the length of curve $\mathcal{C}$ and $s$ its arclength parameterization (in the rest of the paper, we shall note $\widetilde{\mathcal{P}}=w+\mathcal{P}$ ). Even though this is the same energy proposed by Caselles et al. [7] and Yezzi et al. [27], in the well-known geodesic active contour model, Cohen and Kimmel choose a different approach for the minimization of $E$. Instead of using an evolution equation as in $[7,18,27]$, they exploit a method capable of building a curve between two points ( $p_{1}$ and $p_{2}$ ) which is the global minimum of $E$ among all the curves joining these points. This minimum is called a minimal path. Their approach is based on the fact that a minimal path $\mathcal{C}$ between $p_{2}$ and $p_{1}$ can be obtained by 'back propagation', that is solving:

$$
\frac{d \mathcal{C}}{d u}(u)=-\nabla U_{p_{1}}(\mathcal{C}(u)) \text { with } \mathcal{C}(0)=p_{2}
$$

The real function $U_{p_{1}}$, called the minimal action map, is defined at each point $q$ of the image domain by: $U_{p_{1}}(q)=\inf \left\{\int_{0}^{L} \widetilde{\mathcal{P}}(C(s)) d s\right\}\left({ }^{6}\right)$, where the inf is taken among

$\overline{{ }^{6} \text { remark: } \int_{0}^{L} \widetilde{\mathcal{P}}}(C(s)) d s=\int_{0}^{1} \widetilde{\mathcal{P}}(C(u))\left|C^{\prime}(u)\right| d u$ 
all curves such that $\mathcal{C}(0)=p_{1}$ and $\mathcal{C}(L)=q$. In order to compute $U_{p_{1}}$, Cohen and Kimmel[13] use the fact (a proof can be found in [6]) that this map is the solution of the Eikonal equation :

$$
\left\|\nabla U_{p_{1}}\right\|=\widetilde{\mathcal{P}} \text { and } U_{p_{1}}\left(p_{1}\right)=0 .
$$

In order to compute minimal paths in two dimensional images, Cohen and Kimmel numerically solved this equation using a marching algorithm introduced by Tsitsiklis in [26] and rediscovered in [24] as the 'fast marching' algorithm.

\section{From global minimal paths to 3D surface extraction}

The method introduced in [13] can easily be extended for the construction of minimal paths between two points in a 3D image [15]. In that case, the formalism given in the previous section is unchanged, except for the fact that the functions $\widetilde{\mathcal{P}}, U_{p_{1}}$ are defined (and $\mathcal{C}$ takes its values) on a 3D domain. The authors of [15] used it to find centerlines in $3 \mathrm{D}$ tubular structures. As in the previous section, the cost function $\widetilde{\mathcal{P}}$ is assumed to have lower values on a surface to be extracted from the 3D image. Before reviewing ideas in $[3,4]$, where the minimal path framework was extended to the extraction of surfaces between two given curves $\Gamma_{1}$ and $\Gamma_{2}$, we give for comparison purposes a brief description of the (now classical) 'geodesic' active surface approach.

\subsection{Background on active surfaces}

The active surface model, introduced in [8], is a variational approach for the segmentation of objects in 3D images. In the same spirit as geodesic active contours, it is based on finding a surface which minimizes an energy of the form:

$$
E_{S}(S)=\int_{O} \mathcal{P}(S)\left\|\frac{\partial S}{\partial u} \wedge \frac{\partial S}{\partial v}\right\| d u d v
$$

where $(u, v)$ are the parameters of $S$, defined on the open set $O \subset \mathbb{R}^{2}$. The most common procedure for finding a local minimum of this energy is to deform, until convergence, an initial surface $S_{0}$, according to the evolution equation:

$$
\frac{\partial S}{\partial t}=\mathcal{P} H N_{S}-\left(\nabla \mathcal{P} . N_{S}\right) N_{S} \text { with } S(\cdot, \cdot, 0)=S_{0},
$$

$H$ being the mean curvature of $S$ and $N_{S}$ its normal. This implies that the final surface is the local minimum of $E_{S}$ which is "closest" to $S_{0}$. As with geodesic active contours, this method lacks robustness with respect to $S_{0}$. In [3] a method was suggested, based on minimal paths, that provided this model with a convenient initialization. This was done by incorporating the information given by the user through the two constraining curves, $\Gamma_{1}, \Gamma_{2}$. We give a description of this approach in the next section since our algorithm, further proposed, elaborates on ideas that are related. 


\subsection{Minimal path set between two curves}

Active surfaces are usually initialized with simple geometric structures like ellipsoids or cylinders which do not always lead to a good segmentation after evolution to a steady state. Here, instead, the user is asked to introduce into the 3D image a couple of curves (not necessarily planar) drawn on the surface to be extracted. These curves, $\Gamma_{1}$ and $\Gamma_{2}$, are exploited as the initialization of the model. The approach is based on considering a network of paths that globally minimizes an energy associated to the image. This network is used to generate a surface that contains the constraining curves and provides a segmentation of the object lying between them.

A curve $\gamma_{\Gamma_{1}}^{q}$ is a path between a point $q$ and curve $\Gamma_{1}$ if $\gamma_{\Gamma_{1}}^{q}(0)=q$ and $\gamma_{\Gamma_{1}}^{q}(L) \in \Gamma_{1}$. A path network $\mathcal{N}_{\Gamma_{1}}^{\Gamma_{2}}$, between the points of $\Gamma_{2}$ and curve $\Gamma_{1}$, is the set

$$
\mathcal{N}_{\Gamma_{1}}^{\Gamma_{2}}=\left\{\gamma_{\Gamma_{1}}^{q}\right\}_{q \in \Gamma_{2}} .
$$

Using the geodesic energy of each path composing the network, we define the following energy on the set of all possibles networks:

$$
E_{N e t}\left(\mathcal{N}_{\Gamma_{1}}^{\Gamma_{2}}\right)=\int_{q \in \Gamma_{2}} \int_{0}^{L(q)} \widetilde{\mathcal{P}}\left(\gamma_{\Gamma_{1}}^{q}(s)\right) d s d q
$$

Since the potential function $\widetilde{\mathcal{P}}$ is positive, the minimization of $E_{N e t}$ can be obtained by finding every globally minimal path between the points of $\Gamma_{2}$ and curve $\Gamma_{1}$. Moreover, these minimal paths are easily found. Indeed, similar to section 2.2, the minimal path between $\Gamma_{1}$ and a point $q$ (further noted $\mathcal{C}_{\Gamma_{1}}^{q}$ ), with respect to the energy $E$ (defined by (2)), is solution of the ordinary differential equation:

$$
\frac{d \mathcal{C}_{\Gamma_{1}}^{q}}{d u}(u)=-\nabla U_{\Gamma_{1}}\left(\mathcal{C}_{\Gamma_{1}}^{q}(u)\right) \text { with } \mathcal{C}_{\Gamma_{1}}^{q}(0)=q .
$$

$U_{\Gamma_{1}}$ is the action map defined on each point $q \in \Omega$ by :

$$
U_{\Gamma_{1}}(q)=\inf \left\{\int_{0}^{L} \widetilde{\mathcal{P}}(\mathcal{C}(s)) d s\right\},
$$

where the inf is taken among all curves such that $C(0)=q$ and $\mathcal{C}(L) \in \Gamma_{1}$. Furthermore, it follows that $U_{\Gamma_{1}}(q)=\inf _{p \in \Gamma_{1}}\left\{U_{p}(q)\right\}$. This implies, as a consequence of relation (3), that $U_{\Gamma_{1}}$ is also a solution to the Eikonal equation but with a different boundary condition :

$$
\left\|\nabla U_{\Gamma_{1}}\right\|=\widetilde{\mathcal{P}}, \text { and } \forall p \in \Gamma_{1}, U_{\Gamma_{1}}(p)=0 .
$$

By solving equation (7), using each point of $\Gamma_{2}$ as part of the initial condition $\left(\mathcal{C}_{\Gamma_{1}}^{q}(0)=\right.$ $q$ ), we globally minimize the energy $E_{N e t}$, producing the minimal energy network:

$$
\mathcal{S}_{\Gamma_{1}}^{\Gamma_{2}}=\bigcup_{q \in \Gamma_{2}}\left\{\mathcal{C}_{\Gamma_{1}}^{q}\right\}
$$


The minimal network is thus the set of all solutions of the ordinary differential equation (7) when varying its initial condition along $\Gamma_{2}$. Up to a reparameterization, assume every minimal path (respectively curve $\Gamma_{2}$ ) is parameterized on an interval $J$ (respectively $I$ ). $\mathcal{S}_{\Gamma_{1}}^{\Gamma_{2}}$ can then be considered as a mapping (since minimal paths cannot cross without merging) from $I \times J$ to $\Omega$, such that for all pair $(u, v) \in I \times J$,

$\mathcal{S}_{\Gamma_{1}}^{\Gamma_{2}}(u, v)=\mathcal{C}_{\Gamma_{1}}^{\Gamma_{2}(u)}(v)$. Using this map for segmentation follows the same intuition as in $[3,4]$, where the hypothesis is made that each path of $\mathcal{S}_{\Gamma_{1}}^{\Gamma_{2}}$ is within a small distance from the surface to extract. Unfortunately, as can be understood from [21], in the general case the map $\mathcal{S}_{\Gamma_{1}}^{\Gamma_{2}}(\cdot, \cdot)$ lacks the fundamental property of continuity. For example, the surface obtained in figure $2 b$ has a hole and does not recover the half-sphere. For that reason, it is insufficient for segmentation. In order to cope with this difficulty, two different solutions were proposed in $[3,4]$ :

- An analytical interpolation method was used to generate a surface from a finite number of paths belonging to $\mathcal{S}_{\Gamma_{1}}^{\Gamma_{2}}$. This approach gave satisfactory segmentation results only in very particular cases (the topology of the surface being such that the gaps created by the discontinuities uncover relatively small areas of the surface), and was thus preferred as an initialization of other active object methods [3].

- A different network was generated by solving a projected version of the ordinary differential equation (7) [4]. The projection was made on planes whose definition depended on $\Gamma_{1}$ and $\Gamma_{2}$. Even though satisfactory results were obtained in medical images under some restrictions applied to the two constraining curves (they should neither intersect nor be open), with this approach the network is no longer minimal for energy $E_{N e t}$. Furthermore, paths can cross without merging thus no mapping can be defined. Last but not least, this approach can only extract surfaces of objects whose topology is cylindrical.

In order to solve the problems mentioned above, we introduce in the next section a novel approach for the generation of a surface using the minimal path network. This surface shall be defined as the zero level set of a function $\Psi$ which solves a certain transport equation.

\section{Implicit definition of a surface containing the minimal path set}

In order to simplify our description, $\Gamma_{1}$ and $\Gamma_{2}$ are assumed to be two non-intersecting planar, closed curves. We look for a real function $\Psi$, defined on the image domain $\Omega$, such that $\mathcal{S}_{\Gamma_{1}}^{\Gamma_{2}}$ is contained in its zero level set $\Psi^{-1}(\{0\})$. Even though this approach (describing our set through the zero level set of a function) seems related to the 'Level Set Method' introduced by Osher and Sethian in [22], the reader shall see that this approach is substantially different.

Having no a priori knowledge on the properties $\Psi$ should satisfy, we shall assume that $\Psi$ is continuously differentiable and we first look for a necessary condition based on our knowledge of the minimal path network. Further, this condition is exploited to formulate a sufficient condition and finally give a consistent description of the function $\Psi$. 


\subsection{Searching for an implicit function}

As in section 3.2, we denote by $\mathcal{C}_{\Gamma_{1}}^{q}$ a minimal path from a point $q \in \Omega$ to the curve $\Gamma_{1}$, and we assume that $J=[0, \beta]$ is its parameterization interval. The minimal paths set $\mathcal{S}_{\Gamma_{1}}^{\Gamma_{2}}$ can also be considered as a subset of $\Omega, p \in \mathcal{S}_{\Gamma_{1}}^{\Gamma_{2}}$ means that $p$ is a point belonging to a minimal path (joining a point of $\Gamma_{2}$ and $\Gamma_{1}$ ). Let us first assume that $\Psi$ is a continuously differentiable function, defined on $\Omega$ such that $\mathcal{S}_{\Gamma_{1}}^{\Gamma_{2}} \subset \Psi^{-1}(\{0\})$. This means that for all minimal paths $\mathcal{C}_{\Gamma_{1}}^{q}$ we have $\forall u \in J, \Psi\left(\mathcal{C}_{\Gamma_{1}}^{q}(u)\right)=0$. From the derivative with respect to $u$ of this relation we obtain

$$
\forall u \in J, \nabla \Psi\left(\mathcal{C}_{\Gamma_{1}}^{q}(u)\right) \cdot \frac{d \mathcal{C}_{\Gamma_{1}}^{q}}{d u}(u)=0 .
$$

Using relation (7) we deduce the following proposition:

Proposition 4.11 (Necessary condition) For any real differential function $\Psi$, defined on $\Omega$, and such that $\mathcal{S}_{\Gamma_{1}}^{\Gamma_{2}} \subset \Psi^{-1}(\{0\})$, we have for every point $p$ of $\mathcal{S}_{\Gamma_{1}}^{\Gamma_{2}}$ :

$$
\nabla \Psi(p) . \nabla U_{\Gamma_{1}}(p)=0 .
$$

The perpendicularity of the two gradient vector fields is only necessary on the points of the minimal path network. Hardening this condition and demanding that $\Psi$ satisfies a relation similar to (10) everywhere in $\Omega$, should lead to a sufficient relation for the minimal paths to be contained in $\Psi^{-1}(\{0\})$.

It is interesting to note that we only want to act on the zero level set of $\Psi$; this gives the opportunity to introduce a regularization term in the previous equation. For this purpose we introduce a regular (at least continuously differentiable) real function $G$ that satisfies $G(0)=0$. We have the following proposition :

Proposition 4.12 (Sufficient condition) If $\Psi$ is a $C^{1}$ function satisfying the two conditions :

$$
\left\{\begin{aligned}
\left(C_{1}\right) \forall p \in \Omega, \nabla \Psi(p) . \nabla U_{\Gamma_{1}}(p)+G \circ \Psi(p) & =0 \\
\left(C_{2}\right) & \forall q \in \Gamma_{2}, \Psi(q)=0
\end{aligned}\right.
$$

then the minimal path network $\mathcal{S}_{\Gamma_{1}}^{\Gamma_{2}}$ is contained in the zero level set of $\Psi$.

Proof: For every point $q \in \Gamma_{2}$, the values taken by function $\Psi$ along the minimal path $C_{\Gamma_{1}}^{q}$ are given by the function $f_{q}=\Psi \circ \mathcal{C}_{\Gamma_{1}}^{q}$. The derivative of $f_{q}$, for all $u \in J$, gives:

$$
\frac{d f_{q}}{d u}(u)=\nabla \Psi\left(C_{\Gamma_{1}}^{q}(u)\right) \cdot \frac{d \mathcal{C}_{\Gamma_{1}}^{q}}{d u}(u) \underbrace{=}_{\text {from }(7)}-\nabla \Psi\left(\mathcal{C}_{\Gamma_{1}}^{q}(u)\right) . \nabla U_{\Gamma_{1}}\left(\mathcal{C}_{\Gamma_{1}}^{q}(u)\right) \underbrace{=}_{\text {from }\left(C_{1}\right)} G\left(f_{q}(u)\right) .
$$

Thus, the function $f_{q}$ satisfies over the interval $J$ the ordinary differential equation

$$
\frac{d f_{q}}{d u}=G\left(f_{q}\right)
$$

Furthermore, recall that $C_{\Gamma_{1}}^{q}(0)=q$ and $q \in \Gamma_{2}$. Condition $\left(C_{2}\right)$ establishes then that $f_{q}(0)=0$. Consequently, since $G$ is a differentiable function such that $G(0)=0$, the 
unique solution to (12) is $f_{q}=0$ on $J$. A simple proof of this can be obtained by denoting $H(u)=\frac{G(u)}{u}$ and $H(0)=G^{\prime}(0)$. Since $G$ is continuously differentiable and $G(0)=0, H$ is well defined and continous. Denoting $H_{1}$ such that $H_{1}^{\prime}(u)=G(u)$ and multiplying equation 12 by $\exp \left(-H_{1}\right)$, we get that the derivative of $\exp \left(-H_{1}\right) f_{q}$ is equal to zero. the latter function is thus constant, and since its value at 0 is zero, we have $f_{q}=0$ on $J$. In other words, the unique value taken by function $\Psi$ along any minimal path originated on a point $q$ of $\Gamma_{2}$ is zero, which is exactly $\mathcal{S}_{\Gamma_{1}}^{\Gamma_{2}} \subset \Psi^{-1}(\{0\})$.

A quick examination of the conditions given in (11) may not be enough to understand why this implicit approach will produce a better segmentation than explicitly generating a finite number of paths of $\mathcal{S}_{\Gamma_{1}}^{\Gamma_{2}}$ followed by interpolation (as in [4]). The following proposition makes this clear by establishing that the zero level set of the solution to (11) has a very particular structure: it is completely composed of globally minimal paths.

Proposition $4.13\left(\Psi^{-1}(\{0\})\right.$ structure) If $\Psi$ satisfies the same conditions as in proposition 4.12, then for all $p \in \Psi^{-1}(\{0\})$, the minimal path $\mathcal{C}_{\Gamma_{1}}^{p}$ joining $p$ to $\Gamma_{1}$ is also included in this zero level set.

Proof: If $\Psi$ satisfies the conditions of proposition 4.12, then for any point $p$ of $\Omega$, function $f_{p}=\Psi \circ C_{\Gamma_{1}}^{p}$ satisfies the ordinary differential equation (12). If $p$ is on the zero level set of $\Psi$ then $f_{p}(0)=\Psi(p)=0$ and, as in the proof of the previous proposition, $\Psi \circ C_{\Gamma_{1}}^{p}=0$. This means that every point of $\Psi^{-1}(\{0\})$ belongs to a minimal path.

Being minimal with respect to the geodesic energy $E$ (see section 2.2), these paths tend to be traced on the object to extract (as $\Gamma_{1}$ and $\Gamma_{2}$ ). This explains the better results, compared to [4], obtained with our method. A good example is given in figure 2, which demonstrates, on a synthetic image, how this approach gives good results where clearly $\mathcal{S}_{\Gamma_{1}}^{\Gamma_{2}}$ is insufficient for segmentation.

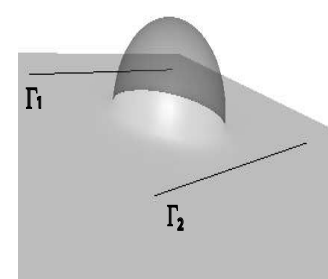

(a)

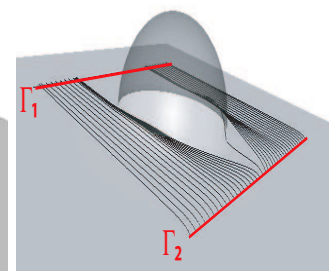

(b)

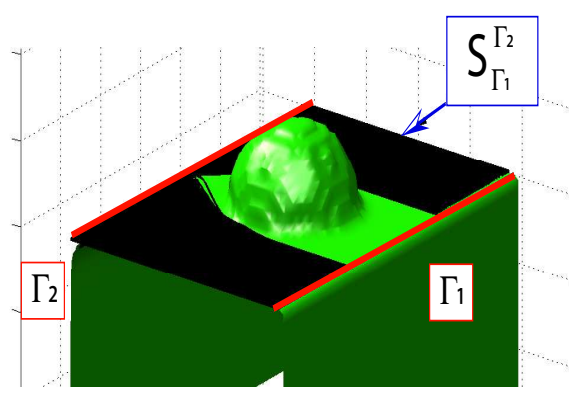

$(c)$

Fig. 2: (a) represents a half-sphere blended with a plane (transparent visualization) and $\Gamma_{1}$ and $\Gamma_{2}$ (black segments). (b) shows some minimal paths of $\mathcal{S}_{\Gamma_{1}}^{\Gamma_{2}}$ taking a short cut around the sphere. (c) shows the superposition of $\Psi^{-1}(0)$ and the set $\mathcal{S}_{\Gamma_{1}}^{\Gamma_{2}}$ (see please the electronic color version). 
In the next sections we explicitly give the problem to be solved in order to build the segmentation of an object from the two constraining curves. Most likely traced by a human user (or a 2D segmentation process), $\Gamma_{1}$ and $\Gamma_{2}$ will be assumed to be planar curves but this is not a necessity.

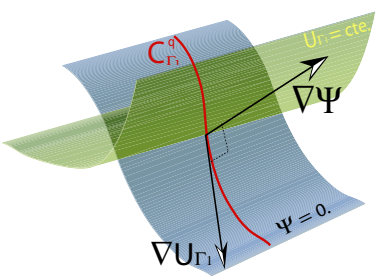

(a) Along a minimal path the gradient of $\Psi$ should be perpendicular to the gradient of the action map.

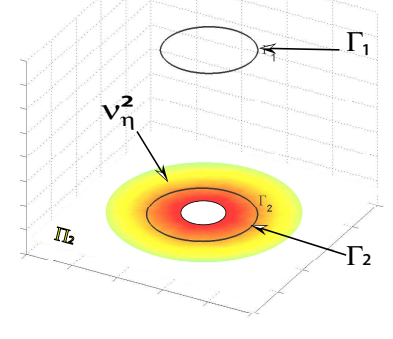

(b) Boundary conditions for the transport problem $\left(\Pi_{1}\right.$ and $\Pi_{2}$ are not necessarily parallel).

Fig. 3:

\subsection{Using minimal paths for surface segmentation}

We denote by $\Pi_{1}, \Pi_{2}$ the intersection of the planes containing $\Gamma_{1}$ and $\Gamma_{2}$ with the image domain (this is purely a practical condition, it is not necessary that these curves are planar but the description of the boundaries of our problem becomes simpler this way). The functions $d_{1}, d_{2}$ are the signed distance functions to these curves, positive in their interior and defined on $\Pi_{1}$ and $\Pi_{2}$ respectively. Notice that at each point $q \in$ $\Gamma_{2}, d_{2}(q)=0$.

Consider now the closed set

$$
\mathcal{V}_{\eta}^{2}=\left\{p \in \Pi_{2} \text { such that }\left|d_{2}(p)\right| \leqslant \eta\right\},
$$

where $\eta$ is a real positive value (see figure 3(b)). Inspired by proposition 4.12, we consider the open set $\mathcal{O}=\operatorname{int}(\Omega)-\mathcal{V}_{\eta}^{2}$, where $\operatorname{int}(\Omega)$ is the interior of the image domain, and search for $\Psi$ as the solution to the Cauchy problem defined on $\Omega$ :

$$
\begin{cases}\nabla \Psi(p) \cdot \nabla U_{\Gamma_{1}}(p)+G \circ \Psi(p)=0 & \text { if } p \in \mathcal{O} \\ \Psi(p)=d_{2}(p) & \text { if } p \in \mathcal{V}_{\eta}^{2} \\ \Psi(p)=\min _{p \in \Pi_{2}}\left(d_{2}(p)\right) & \text { if } p \in \delta \Omega\end{cases}
$$


$\delta \Omega$ is the boundary of the image domain $\Omega$. Notice that outside $\Gamma_{2}$, the signed distance $d_{2}$ is negative, and thus the minimum value taken in the third equation of (14) is the largest distance to $\Gamma_{2} \Pi_{2}$ with a minus sign. This equation is a stationary transport problem where the function $G$ stands for the source term. It is beyond the scope of this paper to present the theoretical details of the existence and uniqueness of the solution to this first order partial differential problem. As a matter of fact, numerical approaches (see section 6) that take in consideration the presence of possible discontinuities of the function $\Psi$ were proposed before a theoretical framework was fully established $[14,25]$. Let us now observe the influence on our problem of the choice of the function $G$.

\subsection{Choice of the Regularization function $G$}

The function $G$ is only required to be continuously differentiable and satisfy $G(0)=0$. It is interesting to examine some possible choices of $G$ and observe its influence on the solution $\Psi$.

- $\mathbf{G}=0$ : With this choice, problem (14) becomes a stationary transport problem. Equation (12) is in this case $\frac{d f_{q}}{d u}=0$, so that for every point $p$ of $\Omega$ function $\Psi \circ C_{\Gamma_{1}}^{p}$ is constant ( $\Psi$ is constant along any minimal path). $\Psi$ 'transports' the values of the boundary $\mathcal{V}_{\eta}^{2} \cup \delta \Omega$ along the minimal paths (the transport problem has been studied from a theoretical point of view see for example [2] and references within, results of existence and uniqueness have been given by Bouchut et. al. in [5] and L. Ambrosio in [2]).

Even though this choice seems the most natural, the resulting $\Psi$ may present discontinuities. By construction, every curve solution of problem (7) (a minimal path) will join curve $\Gamma_{1}$. If $p$ and $q$ are two points of $\mathcal{V}_{\eta}^{2}$ such that $d_{2}(p) \neq d_{2}(q)$ then along each of the paths $C_{\Gamma_{1}}^{p}$ and $C_{\Gamma_{1}}^{q}, \Psi$ will take these two different values. This assumes that the different values transported by $\Psi$ from the boundaries will 'collapse' on curve $\Gamma_{1}$ producing discontinuities.

- $\mathbf{G} \circ \boldsymbol{\Psi}=\alpha . \boldsymbol{\Psi}: \alpha$ is assumed to be a positive constant. With this choice, equation (12) becomes $\frac{d f_{q}}{d u}=-\alpha f_{q}$, whose solution is $f_{q}(u)=\Psi(q) \exp (-\alpha u)$. Along minimal paths, function $\Psi$ is no longer constant, its value decreases in a exponential manner. It is possible to regularize our problem without the necessity of increasing the degree of our differential equation. In our implementation, $\alpha$ is still a parameter but automatic approaches to find this constant are being studied.

\section{Symmetrization and generalization to more than two constraining curves}

The generation of our minimal path surface by solving problem (14) is not a symmetric process with respect to the two constraining curves. From the definition of the set of minimal paths $\mathcal{S}_{\Gamma_{1}}^{\Gamma_{2}}$ (equation (9)), it is clear that the two constraining curves do not play the same role in the construction of the implicit surface $\Psi^{-1}(\{0\})$. This asymmetry is undesirable, it introduces the ambiguity of two different segmentations for the same 
input (an image and two constraining curves).

Our method for a symmetrical construction uses information coming from both sets of minimal paths $\mathcal{S}_{\Gamma_{1}}^{\Gamma_{2}}$ and $\mathcal{S}_{\Gamma_{2}}^{\Gamma_{1}}\left({ }^{7}\right)$ by exploiting both action maps $U_{\Gamma_{1}}$ and $U_{\Gamma_{2}}{ }^{8}$ ). As before, the segmentation surface will be obtained as the zero level set of a function $\Psi$, solution to a stationary transport problem of the form:

$$
\begin{cases}\nabla \Psi(p) \cdot \nabla U(p)+G \circ \Psi(p)=0 & \text { if } p \in \mathcal{O} \\ \Psi(p)=d_{1}(p) & \text { if } p \in \mathcal{V}_{\eta}^{1} \\ \Psi(p)=d_{2}(p) & \text { if } p \in \mathcal{V}_{\eta}^{2} \\ \Psi(p)=\min _{p \in \Pi_{2} \cup \Pi_{1}}\left(d_{1}(p), d_{2}(p)\right) & \text { if } p \in \delta \Omega\end{cases}
$$

where the set $\mathcal{V}_{\eta}^{1}$ is build as $\mathcal{V}_{\eta}^{2}$ in (13), replacing $\Pi_{2}$ by $\Pi_{1}$ and $d_{2}$ by $d_{1}, \mathcal{O}=\operatorname{int}(\Omega)-$ $\mathcal{V}_{\eta}^{1} \cup \mathcal{V}_{\eta}^{2}, U=\mathcal{G}\left(U_{\Gamma_{1}}, U_{\Gamma_{2}}\right)$ and $\mathcal{G}$ is a two-variable real function that has to be chosen correctly in order to obtain a segmentation of the image, $\mathcal{G}$ should naturally satisfy $\forall(x, y) \mathcal{G}(x, y)=\mathcal{G}(y, x)$ The set $\mathcal{V}_{\eta}^{1}$ is build as $\mathcal{V}_{\eta}^{2}$ in (13), replacing $\Pi_{2}$ by $\Pi_{1}$ and $d_{2}$ by $d_{1}$. We shall consider the particular case of $\mathcal{G}=\max$ (the maximum value). We show that this choice arises from the observation of minimal paths, it is equivalent to exploiting $\mathcal{S}_{\Gamma_{1}}^{\Gamma_{2}}$ and $\mathcal{S}_{\Gamma_{2}}^{\Gamma_{1}}$ in two different regions that constitute a partition of the image domain $\Omega$.

This symmetrization is a fundamental step to the generalization of our approach for more than two constraining curves. We shall see somewhat surprisingly that to constrain our surface with more than two curves we can still solve the same transport problem, only the function $U$ has to be modified.

\subsection{Combining actions maps with the maximum function}

In this section we note $U=\max \left(U_{\Gamma_{1}}, U_{\Gamma_{2}}\right)$ and describe how the solution to the transport problem (15) is related to the sets of minimal paths $\mathcal{S}_{\Gamma_{1}}^{\Gamma_{2}}$ and $\mathcal{S}_{\Gamma_{2}}^{\Gamma_{1}}$.

Partitioning the image domain $\Omega$ Let us consider three subsets of the open domain $\Omega$ :

$$
\begin{gathered}
\mathcal{R}_{12}=\left\{p \in \Omega \mid U_{\Gamma_{1}}(p)<U_{\Gamma_{2}}(p)\right\} \\
\mathcal{R}_{21}=\left\{p \in \Omega \mid U_{\Gamma_{2}}(p)<U_{\Gamma_{1}}(p)\right\} \\
\mathcal{S}_{U_{\Gamma_{1} \Gamma_{2}}}=\left\{p \in \Omega \mid U_{\Gamma_{1}}(p)=U_{\Gamma_{2}}(p)\right\}
\end{gathered}
$$

These regions correspond to the weighted Voronoi partition of $\Omega$ with respect to the constraining curves $\Gamma_{1}$ and $\Gamma_{2}$, weighted by potential $\widetilde{\mathcal{P}}$. If a point $p \in \Omega$ is in $\mathcal{R}_{12}$ then the minimal path (with respect to the cost function (2)) joining $p$ to $\Gamma_{1}$ has a smaller cost than the minimal path joining $p$ to $\Gamma_{2}$. In figures 4(a), 4(b) we give an illustration of

\footnotetext{
${ }^{7} \mathcal{S}_{\Gamma_{2}}^{\Gamma_{1}}$ is defined as the set of minimal paths between the points of curve $\Gamma_{1}$ and $\Gamma_{2}$, symmetrical to (9).

${ }^{8}$ Recall that for any curve $\Gamma$, we refer by $U_{\Gamma}$ to the (viscosity) solution of the Eikonal equation: $\left\|\nabla U_{\Gamma}\right\|=\mathcal{P}$, and $\forall q \in \Gamma, U_{\Gamma}(p)=0$.
} 
these different sets on a synthetic image of a 3D S-shaped tube, where we have chosen $\mathcal{P}=\left(1+|\nabla I|^{2}\right)^{-1}$ so that regions of the image where its gradient is high are favored. Note that the set $\mathcal{S}_{U_{\Gamma_{1} \Gamma_{2}}}$ (the 'isodistance' surface) is not necessarily a plane.

The following proposition shows that the zero level set of the solution to problem (15) is (similar to property 4.13) generated by minimal paths.

Proposition 5.11 Let $\Psi$ be solution to the transport problem

$$
\left\{\begin{array}{l}
\nabla \Psi . \nabla U+G \circ \Psi=0, \\
\Psi_{\mid \mathcal{V}_{\eta}^{1}}=d_{1}, \Psi_{\mid \mathcal{V}_{\eta}^{2}}=d_{2} \\
\Psi_{\mid \delta \Omega}=\min \left(d_{1}, d_{2}\right)
\end{array} \quad \text { with } U=\max \left(U_{\Gamma_{1}}, U_{\Gamma_{2}}\right),\right.
$$

(where $\Psi_{\mid \nu}$ refers to the restriction of the function $\Psi$ to the domain $\nu$ ), for almost every $p \in \Omega-\mathcal{S}_{U_{\Gamma_{1} \Gamma_{2}}}$, if $p$ belongs to $\Psi^{-1}(\{0\}) \cap \mathcal{R}_{21}$ (resp. $\mathcal{R}_{12}$ ), then all the points belonging to the minimal path $C_{\Gamma_{1}}^{p}$ (resp. $C_{\Gamma_{2}}^{p}$ ) joining $p$ to $\Gamma_{1}$ (resp. $\Gamma_{2}$ ) and inside region $\mathcal{R}_{21}$ (resp. $\mathcal{R}_{12}$ ), also belong to the zero level set of $\Psi$. In a more compact manner, this can be written:

$$
p \in \mathcal{R}_{i j} \cap \Psi^{-1}(\{0\}) \Rightarrow\left(C_{\Gamma_{j}}^{p} \cap \mathcal{R}_{i j}\right) \subset \Psi^{-1}(\{0\}) \text { with }\{i, j\} \subset\{1,2\}, i \neq j
$$

Proof: Let $p$ be a point of the zero level set of $\Psi$ belonging to $\mathcal{R}_{21}\left({ }^{9}\right)$. As shown in the proof of proposition 4.12 , the function $f_{p}=\Psi \circ C_{\Gamma_{1}}^{p}$ satisfies

$$
\frac{d f_{p}}{d u}(u)=-\nabla \Psi\left(C_{\Gamma_{1}}^{p}(u)\right) \cdot \nabla U_{\Gamma_{1}}\left(C_{\Gamma_{1}}^{p}(u)\right)
$$

Furthermore, if $p \in \mathcal{R}_{21}$ then there exists an interval $K=[0, \beta] \subset J$ such that $\forall u \in$ $K, C_{\Gamma_{1}}^{p}(u) \in \mathcal{R}_{21}$ (because of the continuity of the minimal path). Thus, by definition, for all $u \in K$,

$$
U_{\Gamma_{1}}\left(C_{\Gamma_{1}}^{p}(u)\right)=\max \left(U_{\Gamma_{1}}\left(C_{\Gamma_{1}}^{p}(u)\right), U_{\Gamma_{2}}\left(C_{\Gamma_{1}}^{p}(u)\right)\right)=U\left(C_{\Gamma_{1}}^{p}(u)\right)
$$

and

$$
\frac{d f_{p}}{d u}(u)=-\nabla \Psi\left(C_{\Gamma_{j}}^{p}(u)\right) \cdot \nabla U\left(C_{\Gamma_{j}}^{p}(u)\right)=G\left(f_{q}(u)\right), \text { with } f_{p}(0)=0
$$

because of the boundary conditions of problem (16). This means that $\forall u \in K, f_{p}(u)=$ $\Psi\left(C_{\Gamma_{1}}^{p}(u)\right)=0$ and thus $\left(C_{\Gamma_{1}}^{p} \cap \mathcal{R}_{21}\right) \subset \Psi^{-1}(\{0\})$.

The zero level set of $\Psi$ is thus generated by minimal paths and both sets $\mathcal{S}_{\Gamma_{1}}^{\Gamma_{2}}$ and $\mathcal{S}_{\Gamma_{2}}^{\Gamma_{1}}$ contribute to its generation in each area of the domain $\Omega:\left(\mathcal{S}_{\Gamma_{1}}^{\Gamma_{2}} \cap \mathcal{R}_{21}\right) \subset \Psi^{-1}(\{0\})$ and $\left(\mathcal{S}_{\Gamma_{2}}^{\Gamma_{1}} \cap \mathcal{R}_{12}\right) \subset \Psi^{-1}(\{0\})$. In figure 4(c) we give an illustration of these sets on the synthetic image of the 3D S-shaped tube, and in figure 5 we show the result obtained on this image when solving problem (16).

\footnotetext{
${ }^{9}$ If $p$ belongs to $\mathcal{R}_{12}$ the proof is identical by interchanging 1 and 2.
} 
In practice, using $U=\max \left(U_{\Gamma_{1}}, U_{\Gamma_{2}}\right)$ has the inconvenience that we lack differentiability on the surface $\mathcal{S}_{U_{\Gamma_{1} \Gamma_{2}}}$. We thus regularize the function $U$ before solving problem (15) by noticing that $\max (x, y)=(x+y+|x-y|) / 2$. The function $\max (x, y)$ is thus smooth (and linear) everywhere except on the line $x=y$. We then replace the absolute value function by a smooth function equal to it except on a small interval around 0 .

\subsection{Minimal path surface constrained by more than two curves}

In this subsection, our aim is to produce an approach capable of taking into account more than two constraining curves. We shall exploit the action maps initialized by each of these curves and a transport problem in order to implicitly generate our $\Psi$ function, whose zero level set contains all the constraining curves and is only composed by minimal paths. As before, the action maps are computed with respect to the potential $\widetilde{\mathcal{P}}$ that gives the information concerning the borders of the object to extract from the image. Our approach can be seen as a generalization of the method proposed in [11] that allows one to connect an unstructured set of points on a 2D image by minimal paths.

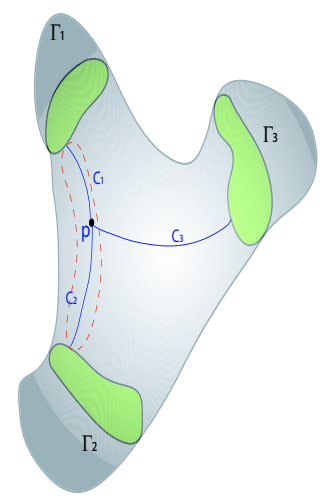

Fig. 6: The couple of minimal paths having the smallest cost show which constraining curves have to be connected to point $p$, in this scheme curves $\Gamma_{1}$ and $\Gamma_{2}$.

by

Let us assume that we are given a set of constraining curves $\left\{\Gamma_{i}\right\}_{1 \leqslant i \leqslant n}$ traced on the object to extract. Consider a point $p \in \Omega$ ( $\Omega$ being the image domain) placed on the surface of the object to be segmented. Consider the $n$ minimal paths $\left\{C_{\Gamma_{i}}^{p}\right\}_{1 \leqslant i \leqslant n}$ (with respect to the cost $E(\mathcal{C})=\int_{\mathcal{C}} \widetilde{\mathcal{P}}$ ) between $p$ and the $n$ constraining curves $\left\{\Gamma_{i}\right\}_{1 \leqslant i \leqslant n}$. Following the approach proposed in [11], it seems natural to assume that on the neighborhood of point $p$ the surface we should generate is only influenced by the two constraining curves that are closer to $p$ with respect to the metric induced by potential $\widetilde{\mathcal{P}}$. These two curves, $\Gamma_{i_{m}}$ and $\Gamma_{j_{m}}$, are those whose associated action maps, $U_{\Gamma_{i_{m}}}(p)$ and $U_{\Gamma_{j_{m}}}(p)$, are the smallest of the set $\left\{U_{\Gamma_{k}}(p)\right\}_{k \in\{1 \ldots n\}}$. On figure 6 we illustrate this approach.

The generalized problem of the multiple constrained minimal path surface, can be reduced locally to the problem of generating a constrained surface with two constraining curves. This situation is identical to the one considered in the previous subsection 5.1, from which we deduce the following formulation: Let $U$ be the real function defined on $\Omega$

$$
\forall p \in \Omega, U(p)=U_{\Gamma_{i_{2}}}(p)
$$

where the index $i_{2}$ is such that $U_{\Gamma_{i_{1}}}(p) \leqslant U_{\Gamma_{i_{2}}}(p) \leqslant \ldots \leqslant U_{\Gamma_{i_{k}}}(p) \leqslant \ldots \leqslant U_{\Gamma_{i_{n}}}(p)$. Note that around any point $p$ we are considering the maximum value of the two smallest action maps, just as if from point $p$ only two curves were seen. 


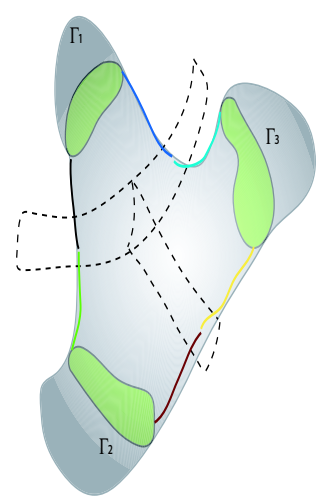

Fig. 7: Partition of $\Omega$ into the weighted Voronoi regions corresponding to the constraining curves.
Consider now the solution $\Psi$ to the transport problem

$$
\left\{\begin{array}{l}
\nabla \Psi . \nabla U+G \circ \Psi=0 \\
\forall i \in\{1 \ldots n\} \Psi_{\mid \mathcal{V}_{\eta}^{i}}=d_{i},
\end{array}\right.
$$

where the sets $\mathcal{V}_{\eta}^{i}$ are the points on the surfaces containing the curves $\Gamma_{i}$ (planes in our examples) and at a distance of at most $\eta . d_{i}$ are the signed distance functions with respect to each constraining curve.

The construction of function the $U$ implies the separation of the set $\Omega$ in $n$ regions $\left\{\mathcal{R}_{i}\right\}_{1 \leqslant i \leqslant n}$. Similar to proposition 5.11 , it can be shown that the zero level set of $\Psi$ is only generated by minimal paths. We produce this result without proof but it is similar to the one of proposition 5.11. Figure 7 illustrates this construction. Note that Deschamps and Cohen treated in $[12,11]$ a similar problem in 3D by linking regions by minimal paths.

In order to illustrate our approach, we show in figure 8 the solution function $\Psi$ and its zero level set obtained on a synthetic image built from an object with a V-shape. Figure 8.a shows three orthogonal slices of this image and the three constraining curves. In figure 8.b we show the separation of the image domain into three Voronoi regions. We have also traced some minimal paths between these curves. On figures 8.c and 8.d we expose, respectively three orthogonal planes of the function $\Psi$, and its zero level set. Note that $\Psi^{-1}(0)$ contains the three constraining curves and extracts the object of interest.

We use the same approach for the segmentation of a torus in figure 9, where we have also shown some minimal paths traced between two of the constraining curves in order to show that they are included in the zero level set of $\Psi$. Notice also that a large part of the torus is not covered by the previous method of path network [4] while our new approach permits a complete segmentation of the surface. 


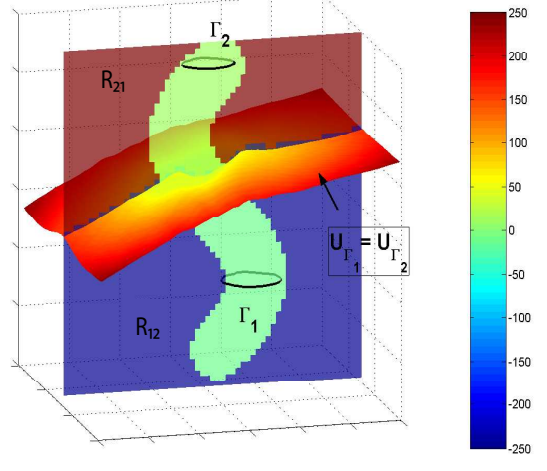

(a) We have colored the points of a plane of this image according to their membership in $\mathcal{R}_{12}, \mathcal{R}_{21}$ or $\mathcal{S}_{U_{\Gamma_{1} \Gamma_{2}}}$.

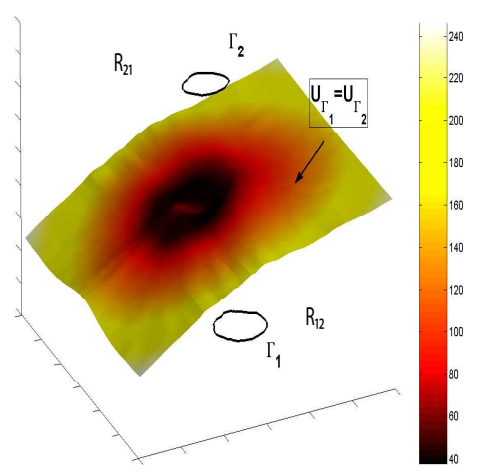

(b) Representation of $\mathcal{S}_{U_{\Gamma_{1} \Gamma_{2}}}$ colored following $U_{\Gamma_{1}}$ values.

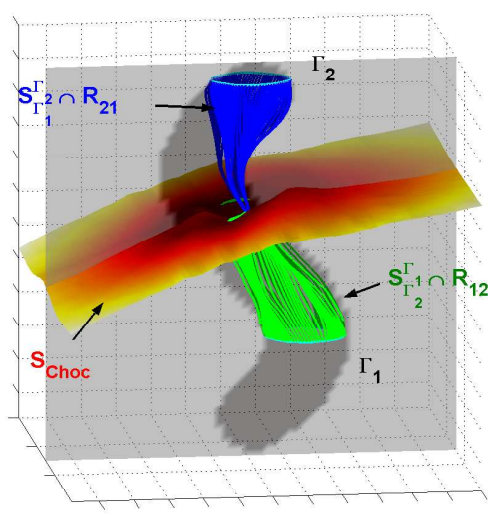

(c) Sets $\mathcal{S}_{\Gamma_{1}}^{\Gamma_{2}} \cap \mathcal{R}_{21}$ and $\mathcal{S}_{\Gamma_{2}}^{\Gamma_{1}} \cap \mathcal{R}_{12}$.

Fig. 4: The synthetic image used for this figure is a binary representation of a 3D S-shaped tube (see also figure 10). 


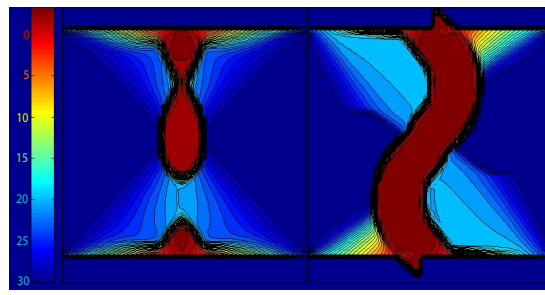

(a)

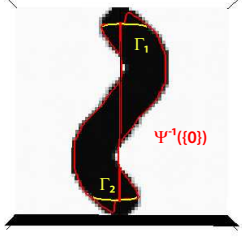

$(b)$

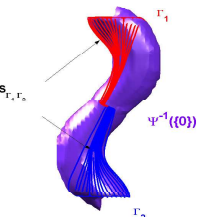

$(c)$

Fig. 5: Solution of the symmetrical transport problem (16) on the 3D S-shaped tube. (a) shows the values taken by $\Psi$ on two orthogonal planes, in (b) we represent a slice of the synthetic image and the zero level set of $\Psi$, in (c) we give a 3D rendering of this zero level set and we have drawn some paths of the sets $\left(\mathcal{S}_{\Gamma_{1}}^{\Gamma_{2}} \cap \mathcal{R}_{21}\right) \subset \Psi^{-1}(\{0\})$ and $\left(\mathcal{S}_{\Gamma_{2}}^{\Gamma_{1}} \cap \mathcal{R}_{12}\right) \subset \Psi^{-1}(\{0\})$.

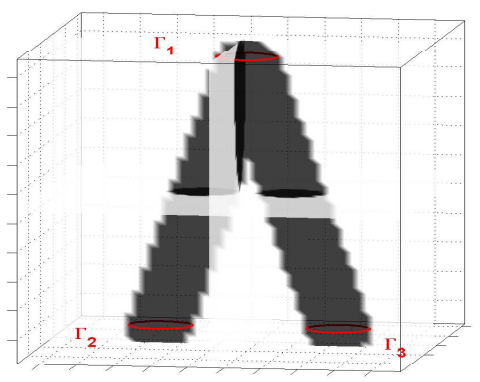

$(a)$

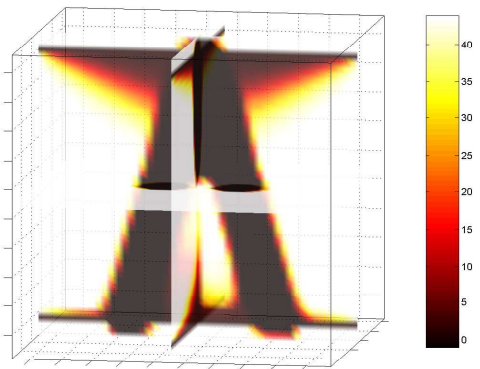

(c)

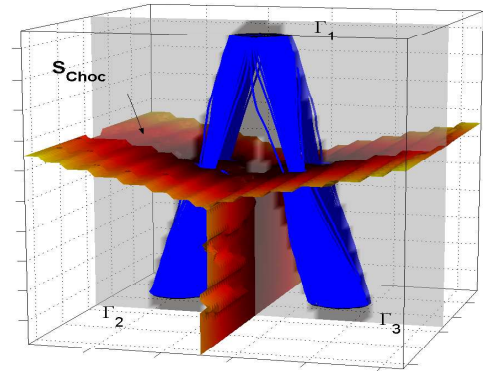

$(b)$

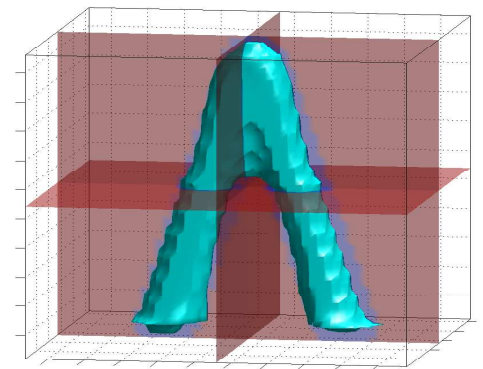

(d)

Fig. 8: Constrained implicit segmentation of a synthetic V-shaped tube under three constraining curves. (a) is the image to segment, (b) shows the Voronoi regions with respect to the curves,(c) shows the values taken by function $\Psi$ on three orthogonal planes and (d) is the final segmentation given by the zero level set of $\Psi$. 


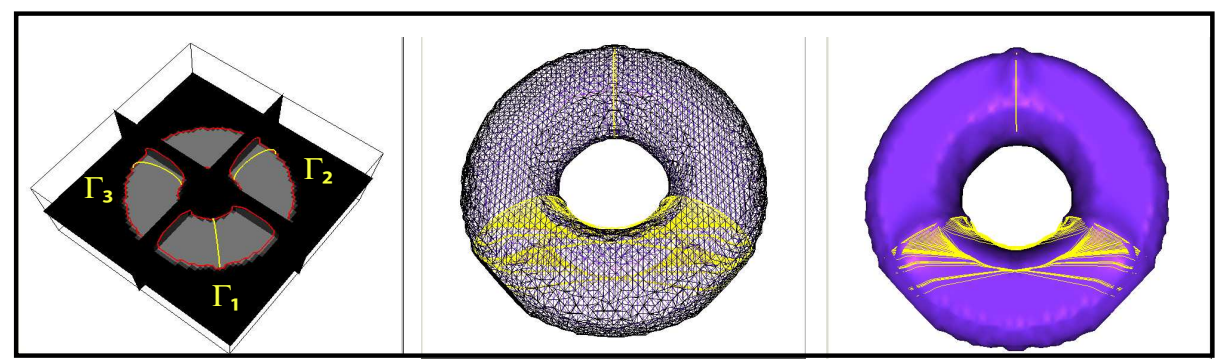

Fig. 9: Implicit segmentation of a torus synthetic image by minimal paths under three constraining curves. We also show some minimal paths between two of the constraining curves to show that they are traced on the zero level set of $\Psi$. 


\section{Implementation Issues}

We now describe an efficient algorithm for the numerical implementation of the transport problem (14) and we assume $G(u)=\alpha u$. Unlike [4], minimal paths are not to be computed directly in this implicit approach. We only numerically calculate solutions to the Eikonal equation and to the stationary transport equations.

To numerically solve the Eikonal equation (8) classic finite difference schemes tend to be unstable. Generally it is preferable to use consistent algorithms using upwind differences (derivative approximations are chosen looking in the direction from which the information is flowing) as fast marching [26, 24] or fast sweeping [17]. Numerical complexity of $O(N \log (N))$ ( $N$ being the number of grid points) can then be achieved and only one grid pass is needed to obtain a first order approximation of the solution $U_{\Gamma_{1}}$. The stationary transport equation, as with most first order partial differential equations whose characteristics intersect, is difficult to solve numerically. In fact, in the general case ( $\widetilde{\mathcal{P}}$ is assumed to be a bounded and continuous function), there is no classical solution defined in all $\Omega$, and the weak solution $\Psi$ can present discontinuities. Many implementations of the transport equation in its non-static expression have been proposed in the modeling of geophysical phenomena. In 1964, Lax and Wendorff proposed in [19] a scheme using centered finite differences for the approximation of derivatives. Then, in 1968, Crowley suggested in [14] a scheme that achieved second order precision in time and space, and which inspired other numerous publications. In particular, a generalization to multiple dimensions was proposed by Smolarkiewicz in [25]. These are only some early publications from a long list of papers treating this topic. Here we will concentrate on a first order, fast algorithm which is less constrained since only the zero level set of the solution matters in our approach.

In order to simplify notation, the symbol $V$ shall be used to refer to the gradient $\nabla U_{\Gamma_{1}}$. One of the first numerical approaches for solving the transport equation proposes a first order approximation of the gradient $\nabla \Psi$ that follows the direction in which information propagates. This discretization is the upwind approach and consists in choosing the approximation of $\frac{\partial \Psi}{\partial \delta}$ following the sign of the components $V_{\delta}$ (where $\delta=x, y$ ou $z$ ) of $V$. Adalsteinsson and Sethian as well as A. Yezzi and J. L. Prince used this scheme in $[1,28]$ for the numerical solution of equation $\nabla \Psi \cdot T=1$ (where $T$ was a known vector field). Lastly, although this scheme is of relatively low precision and dissipative [20], it gives satisfactory results in our experiments with an acceptable convergence speed.

If $\Psi^{i, j, k}$ is the value of the numerical approximation of $\Psi$ at point $[i ; j ; k]$ of the discrete square grid, we shall denote the left and right approximations of the partial derivatives by:

$$
D^{-x} \Psi=\frac{\Psi^{i, j, k}-\Psi^{i-1, j, k}}{h}, D^{+x} \Psi=\frac{\Psi^{i+1, j, k}-\Psi^{i, j, k}}{h}
$$

(similarly in the $y$ and $z$ directions) where $h$ is the discretization step, identical in all three spatial directions. Our scheme for solving the stationary transport problem $V . \nabla \Psi+\alpha \Psi=0$ is then

$$
\begin{aligned}
V_{x}^{i, j, k} \cdot\left(D^{-x} \Psi^{i, j, k} \text { or } D^{+x} \Psi^{i, j, k}\right)+V_{y}^{i, j, k} \cdot\left(D^{-y} \Psi^{i, j, k} \text { or } D^{+y} \Psi^{i, j, k}\right)+ \\
V_{z}^{i, j, k} \cdot\left(D^{-z} \Psi^{i, j, k} \text { or } D^{+z} \Psi^{i, j, k}\right)=-\alpha \Psi^{i, j, k} .
\end{aligned}
$$


where the value of the vector $V$ at grid point $(i, j, k)$ is $\left(V_{x}^{i, j, k}, V_{y}^{i, j, k}, V_{z}^{i, j, k}\right)$. In our problem, the direction in which information propagates is given by the vector $-V$. Therefore, denoting by $H$ the heaviside function defined by $H(x)=\left\{\begin{array}{l}1, \text { if } x \geqslant 0 \\ 0, \text { else. }\end{array}\right.$, the upwind approximation is:

$$
\begin{aligned}
& V_{x}^{i, j, k} \cdot\left(D^{-x} \Psi^{i, j, k} H\left(-V_{x}^{i, j, k}\right)+D^{+x} \Psi^{i, j, k} H\left(V_{x}^{i, j, k}\right)\right)+ \\
& V_{y}^{i, j, k} \cdot\left(D^{-y} \Psi^{i, j, k} H\left(-V_{y}^{i, j, k}\right)+D^{+y} \Psi^{i, j, k} H\left(V_{y}^{i, j, k}\right)\right)+ \\
& V_{z}^{i, j, k} \cdot\left(D^{-z} \Psi^{i, j, k} H\left(-V_{z}^{i, j, k}\right)+D^{+z} \Psi^{i, j, k} H\left(V_{z}^{i, j, k}\right)\right)=-\alpha \Psi^{i, j, k} .
\end{aligned}
$$

Then, denoting $I=(i+1)$ if $V_{x}>0$, and $i-1$ otherwise, and similarly for $J$ and $K$, we have

$$
\begin{gathered}
\left|V_{x}^{i, j, k}\right|\left[\Psi^{I, j, k}-\Psi^{i, j, k}\right]+\left|V_{y}^{i, j, k}\right|\left[\Psi^{i, J, k}-\Psi^{i, j, k}\right] \\
+\left|V_{z}^{i, j, k}\right|\left[\Psi^{i, j, K}-\Psi^{i, j, k}\right]=-\alpha \Psi^{i, j, k}
\end{gathered}
$$

which, by grouping terms with $\Psi^{i, j, k}$, finally leads to the update expression of our algorithm:

$$
\Psi^{i, j, k}=\frac{\left|V_{x}^{i, j, k}\right| \Psi^{I, j, k}+\left|V_{y}^{i, j, k}\right| \Psi^{i, J, k}+\left|V_{z}^{i, j, k}\right| \Psi^{i, j, K}}{\left|V_{x}^{i, j, k}\right|+\left|V_{y}^{i, j, k}\right|+\left|V_{z}^{i, j, k}\right|-\alpha} .
$$

This equality can be exploited, as presented in [28], in a fast marching type scheme that achieves a first order approximation of the solution to our problem in only one grid pass and with a $N \log (N)$ complexity. At the end, our algorithm consists of solving the Eikonal equation first, then the transport equation by means of the same implementation. We thus can achieve very rapid computing times. In the next section we give some results.

Finally, we give a summary of the algorithm we use for the different versions of our method given constraint curves $\Gamma_{i}$ :

1. Compute Minimal action map $U_{\Gamma_{i}}$, by Fast Marching algorithm.

2. Compute $U$ as defined in the previous sections as either $U_{\Gamma_{1}}$ or as a function of the $U_{\Gamma_{i}}$.

3. Numerically Solve Transport problem (14) or (15) or (17) as described in this section in order to find the function $\Psi$.

4. Detection of the zero level set $\Psi^{-1}(0)$ by the Marching Cube algorithm,

5. This surface can be the final result or the function $\Psi$ can be used as initial value for a level set active surface.

\section{Applications}

We apply our method to some synthetic and real 3D images. In all our examples we used a potential of the form: $\widetilde{\mathcal{P}}=\rho \cdot h_{1}\left(\left|\nabla I_{\sigma}\right|\right)+(1-\rho) \cdot h_{2}\left(\Delta I_{\sigma}\right)$, where $h_{1}$ and $h_{2}$ are two functions bounded between 0 and 1 and where $I_{\sigma}$ is the convolution of the given image with a Gaussian kernel of variance $\sigma$. Typically, $h_{1}(x)=\frac{1}{1+x^{2} / \lambda^{2}}$, where $\lambda$ is a user defined contrast factor that can be computed as an average gradient value, and $h_{2}$ is chosen to be a zero crossing detector. 
Figure 2 represents a sphere blended with a plane. This surface is to be extracted between two curves which are parallel lines (see figure 2.a). This configuration does not exactly satisfy the hypothesis taken in section 3 (we are not dealing with closed curves) but the extension is straightforward (the boundary conditions have to be slightly modified). The set of minimal paths $\mathcal{S}_{\Gamma_{1}}^{\Gamma_{2}}$ is unable to provide enough information for the extraction of the surface, since no minimal path 'climbs' on the sphere surface. Nonetheless, the zero level set of the corresponding $\Psi$ function reconstructs perfectly the surface. Our implicit method recovers more information than the minimal paths and we obtain the complete surface.

Figure 10 illustrates with a synthetic binary volume the behavior of our algorithm when various local minima of energy $E_{S}(4)$ are present. In this volume three ' $S$ ' shaped tubes are displayed one inside the other. The constraining curves are traced on the second tube. Without the information they bring, segmenting this tube is a hard task.

In figure 11 we show the extraction of the surface of the left ventricle from the 3D ultrasound image shown in figure 1 . For this ultrasound image of size $256 \times 256 \times$ 256 we used a personal computer with a $1.4 \mathrm{Ghz}$ processor and $512 \mathrm{Mb}$ of RAM. The segmentation was obtained in less than 15 seconds.

In our last example, shown in figure 12 , we display the segmentation of a left ventricle. In this case the information given by the two constraining curves, associated to the fact that the surface is composed of globally minimal paths, is crucially important, since the echogenicity of the patient generates a very low visibility.

\section{Conclusion}

In this paper we have presented a method that generalizes globally minimal paths for curve segmentation in $2 \mathrm{D}$ to surface segmentation in 3D. Our model is initialized by two or more user-supplied curves which we maximally exploit, partly by the fact that the surface we generate is constrained to contain them. We have developed a novel implicit approach that, through a linear partial differential equation, exploits the solution to the Eikonal equation and generates a function whose zero level set is only composed by minimal paths. Hence, our approach is not prone to local minima traps as are other active surface approaches. It is especially well suited for medical image segmentation, in particular for ultrasound image segmentation. In cases where the image quality is very poor, our approach handles the introduction of additional information coming from the practitioner in a very natural manner: a few $2 \mathrm{D}$ segmentations can be enough to generate a coherent, complete surface. 


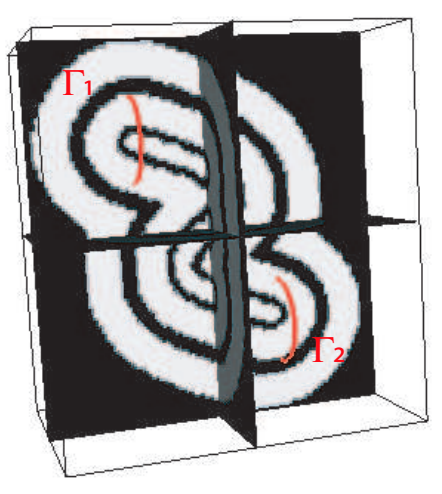

$(a)$

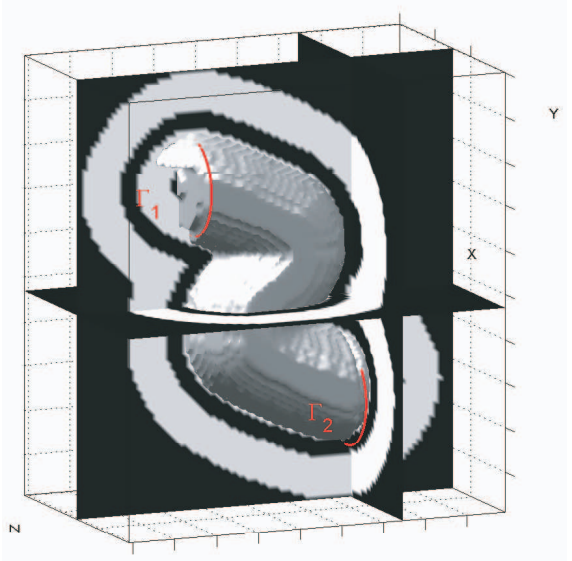

$(c)$

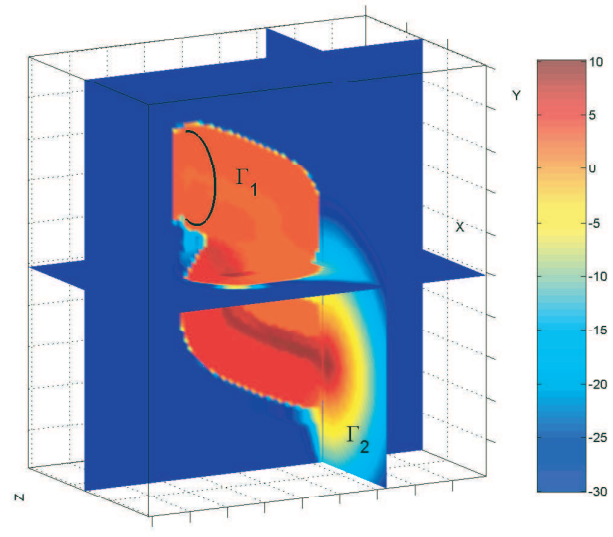

$(b)$

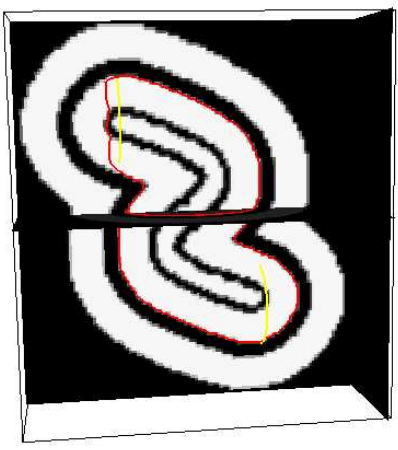

$(d)$

Fig. 10: (a) shows the intersection of a plane with a binary image where three 'S' shaped tubes are one inside the other, $\Gamma_{1}$ and $\Gamma_{2}$ are shown in red. (b) shows the values taken by $\Psi$ on three perpendicular planes (see please the electronic color version). (c) shows the superposition of $\Psi^{-1}(0)$ and the original image and (d) the intersection of this surface with a plane of the image. 


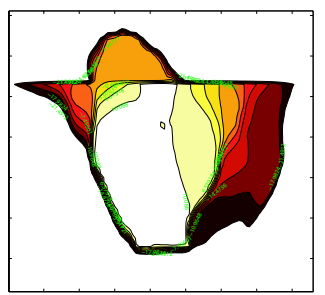

$(a)$

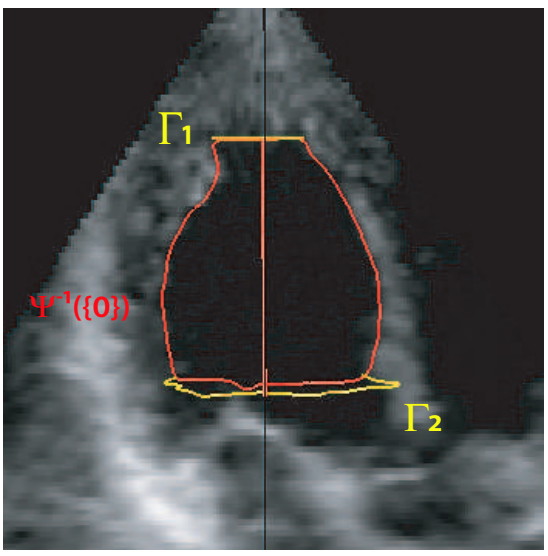

$(d)$

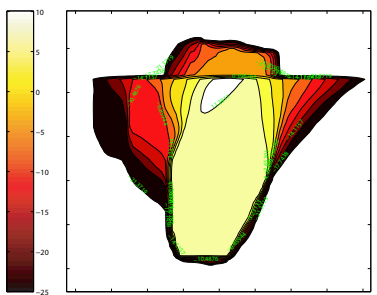

$(b)$

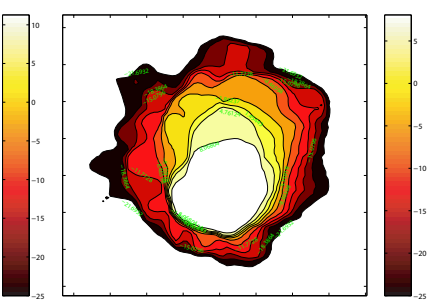

$(c)$

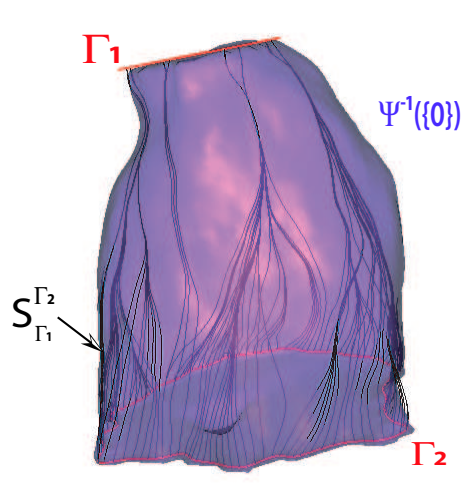

$(e)$

Fig. 11: Left ventricle segmentation : (a),(b) and (c) display some level sets of our solution $\Psi$ on three orthogonal planes. (d) shows the intersection of the zero level set of $\Psi$ with a slice of the image and (e) shows a volume representation of $\Psi^{-1}(\{0\})$ (see please the electronic color version). 


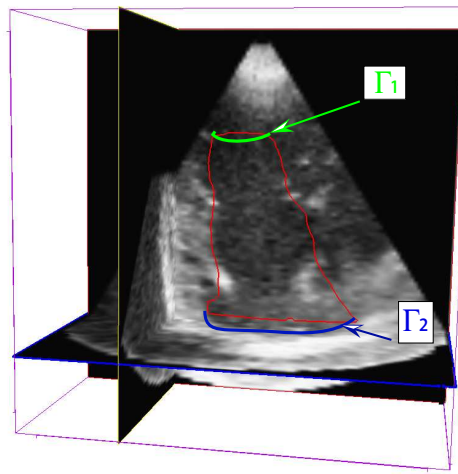

$(a)$

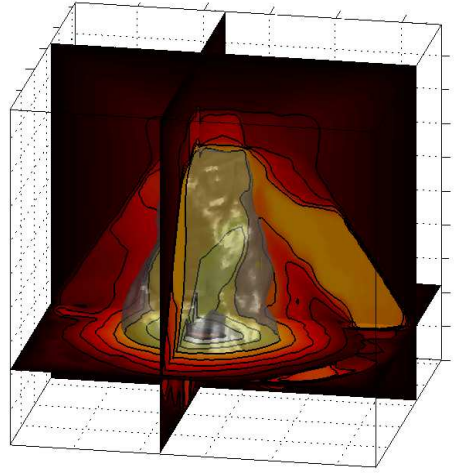

(b)

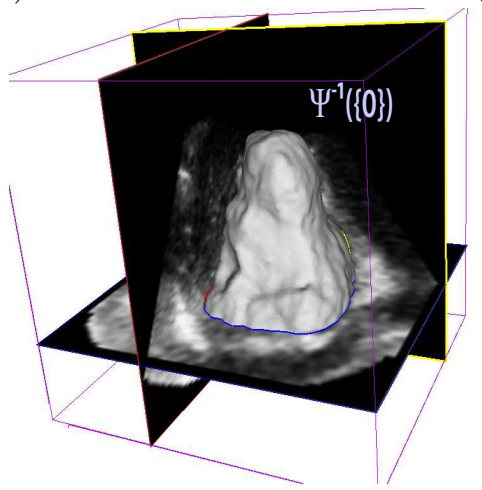

(c)

Fig. 12: (a) shows a plane of a 3D ultrasound volume obtained from a patient whose echogenicity is low. This image is difficult to segment. (b) shows some level sets of our solution $\Psi$ and a volume representation of the zero level set. (c) displays the segmentation obtained with our method(see please the electronic color version). 


\section{References}

1. D. Adalsteinsson and J.A. Sethian. The fast construction of extension velocities in level set methods. Journal of Computational Physics, 148:2-22, 1999.

2. L. Ambrosio. Transport equation and Cauchy problem for BV vector fields. Preprints Scuola Normale Superiore, Department of Mathematics: http://cvgmt.sns.it/people/ambrosio/, 2003.

3. R. Ardon and L.D. Cohen. Fast constrained surface extraction by minimal paths. 2nd IEEE Workshop on Variational, Geometric and Level Set Methods in Computer Vision, pages 233244, October 2003.

4. R. Ardon and L.D. Cohen. Fast Constrained Surface Extraction by Minimal Paths. To appear in International Journal of Computer Vision, 2006.

5. F. Bouchut, F. James, and S. Mancini. Uniqueness and weak stability for multi-dimensional transport equations with one-sided lipschitz coefficient. Prépublications du département Mathématiques et Applications, Physique Mathématique d'Orléans, 2004.

6. A.M. Bruckstein. On shape from shading. Computer Vision, Graphics and Image Processing, 44(2):139-154, November 1988.

7. V. Caselles, R. Kimmel, and G. Sapiro. Geodesic active contours. International Journal of Computer Vision, 22(1):61-79, 1997.

8. V. Caselles, R. Kimmel, G. Sapiro, and C. Sbert. Minimal-surfaces based object segmentation. IEEE Transactions On Pattern Analysis and Machine Intelligence, 19(4):394-398, April 1997.

9. L.D. Cohen. On active contour models and balloons. CVGIP, 53(2):211-218, 1991.

10. L.D. Cohen. Avoiding local minima for deformable curves in image analysis. In Curves and Surfaces with Applications in CAGD, Nashville, 1997. Vanderbilt Univ. Press.

11. L.D. Cohen. Multiple contour finding and perceptual grouping using minimal paths. Journal of Mathematical Image Vision, 14(3), 2001. Jan 2001.

12. L.D Cohen and T. Deschamps. Grouping connected components using minimal path techniques. application to reconstruction of vessels in 2D and 3D images. Proceedings of the IEEE Computer Society Conference on Computer Vision and Pattern Recognition(CVPR'01), 2001.

13. L.D. Cohen and R. Kimmel. Global minimum for active contour models: A minimal path approach. International Journal of Computer Vision, 24(1):57-78, August 1997.

14. W. P. Crowley. Numerical advection experiments. Monthly Weather Review, 96:1-11, 1968.

15. T. Deschamps and L.D. Cohen. Fast extraction of minimal paths in 3D images and applications to virtual endoscopy. Medical Image Analysis, 5(4), December 2001.

16. O. Gerard, T. Deschamps, M. Greff, and L. D. Cohen. Real-time interactive path extraction with on-the-fly adaptation of the external forces. European Conference on Computer Vision, june 2002.

17. C. Y. Kao, S. Osher, and J. Qian. Lax-Friedrichs sweeping scheme for static Hamilton-Jacobi equations. Journal of Computational Physics, 26:367-391, Mai 2004.

18. M. Kass, A. Witkin, and D. Terzopoulos. Snakes: Active contour models. International Journal of Computer Vision, 1(4):321-331, 1988.

19. P. D. Lax and B. Wendorff. Systems of conservation laws. Communications on Pure and Applied mathematics, 17:381-398, 1964.

20. R. J. LeVeque. High-resolution conservative algorithms for advection in incompressible flow. SIAM Journal on Numerical Analysis, 33(2):627-665, April 1996.

21. C. Mantegazza and A. C. G. Mennucci. Hamilton-Jacobi equations and distance functions on Riemannian manifolds. Appl. Math. Opt., 47(1):1-25, 2003.

22. S. Osher and J.A. Sethian. Fronts propagating with curvature dependent speed: algorithms based on the hamilton-jacobi formulation. Journal of Computational Physics, 79:12-49, 1988. 
23. N. Paragios. Geodesic Active Regions and Level Set Methods: Contributions and Applications in Artifi cial Vision. PhD thesis, Université de Nice Sophia-Antipolis, France, 2000.

24. J.A. Sethian. A fast marching level set method for monotonically advancing fronts. Proceedings of the National Academy of Sciences, 93(4):1591-1595, February 1996.

25. P. K. Smolarkiewicz. The multi-dimensional crowley advection scheme. Monthly Weather Review, 110:1968-1983, 1982.

26. J. N. Tsitsiklis. Efficient algorithms for globally optimal trajectories. IEEE Transactions on Automatic Control, 40(9):1528-1538, September 1995.

27. A. Yezzi, S. Kichenassamy, A. Kumar, P. Olver, and A. Tannenbaum. A geometric snake model for segmentation of medical imagery. IEEE Transactions On Medical Imaging, 16(2):199-209, Avril 1997.

28. A. Yezzi and J. L. Prince. An Eulerian PDE Approach for Computing Tissue Thickness. IEEE Transactions On Medical Imaging, 22:1332-1339, October 2003.

29. A.L. Yuille, P.W. Hallinan, and D.S. Cohen. Feature extraction from faces using deformable templates. International Journal of Computer Vision, 8(2):99-111, August 1992.

30. S.C. Zhu and A. Yuille. Region competition: Unifying snakes, region growing, and bayes/mdl for multiband image segmentation. IEEE Transactions On Pattern Analysis and Machine Intelligence, 18(9):884-900, September 1996.

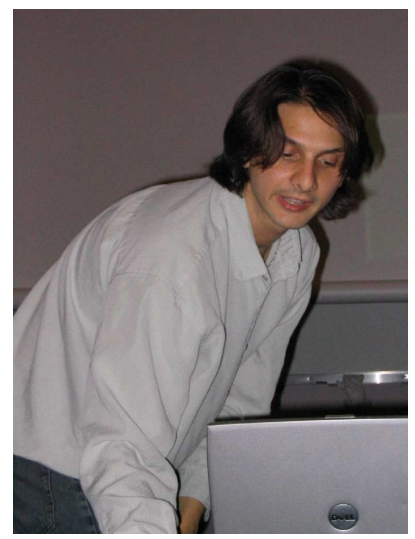

Roberto Ardon graduated from the Ecole Centrale Paris in 2001 with a major in applied mathematics, obtained his master degree in image processing from the Ecole Normale Supérieure de Cachan in the same year and his Ph.D. degree in applied mathematics from the University Paris-Dauphine in 2005. Currently he is a research scientist in Philips Medical Systems Research Paris. His research interests include calculus of variations mainlly focused on medical image processing.

Laurent D. Cohen was at Ecole Normale Superieure Ulm in Paris from 1981 to 1985. He received Master's and Ph.D. degrees in Applied Mathematics from Paris 6 in 1983 and 1986. From 1985 to 1987, he was member at the Computer Graphics and Image Processing group at Schlumberger Palo Alto Research, California and Schlumberger Montrouge Research, and remained consultant there for a few years afterwards. He began working with INRIA, France in 1988, mainly with the medical image understanding group Epidaure. Since 1990, he is Research Scholar (Charge then Directeur 


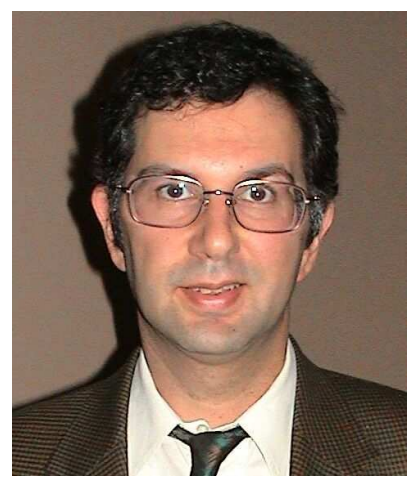

de Recherche) with CNRS in the Applied Mathematics and Image Processing group at CEREMADE, University Paris-Dauphine. His research interests and teaching at the university are applications of variational methods and Partial Differential Equations to Image Processing and Computer Vision, like deformable models, minimal paths, surface reconstruction, Image registration, Image segmentation and restoration. He obtained CS 2002 Prize for Image and Signal Processing. He has been member in program committees for boards for about 20 international conferences.

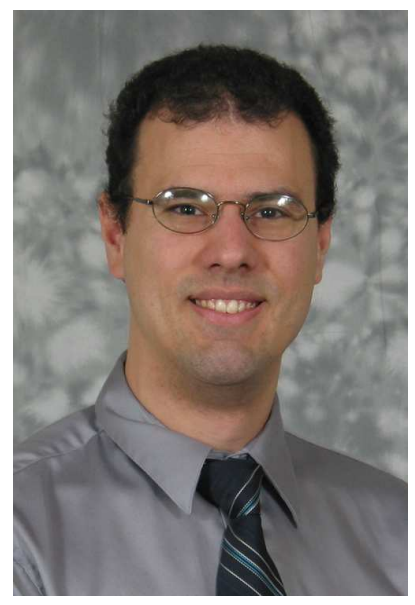

Anthony Yezzi obtained his Ph.D. in 1997 through the Department of Electrical Engineering at the University of Minnesota. After completing a postdoctoral research position in the Laboratory for Information and Decision Systems (LIDS) at Massacusetts Institute of Technology, he joined the faculty of the School of Electrical and Computer Engineering at Georgia Institute of Technology in 1999 where he currently holds the position of Associate Professor. Prof. Yezzi has also consulted for a number of medical imaging companies including GE, Picker, and VTI, and has been an IEEE member since 1999. His research lies primarily within the fields of image processing and 
computer vision. He has worked on a variety of problems including image denoising, edge-detection, segmentation and grouping, shape analysis, multi-frame stereo reconstruction, tracking, and registration. Some central themes of his research include curve and surface evolution theory, differential geometry, and partial differential equations. 